\title{
An Accurate Interference Refractometer Based on a Permanent Vacuum Chamber - Development and Results
}

\author{
M. J. Renkens, P. H. Schellekens (2), Eindhoven University of Technology/Netherlands \\ Received on January 15, 1993
}

SUMMARY

\begin{abstract}
A new accurate interference refractometer, without pumping device. has been developed. The refractometer is based on a commercially available laser measurement system and uses a prismatic measuring cell, containing a permanent vacuum chamber. Measurements of the refractive index of air can be carried out accurately, easily and quickly with this instrument. For this instrument also a new arrangement of a double pass, plane mirror interferometer with high stability was developed. The accuracy of the interference refractometer is analytically and experimentally determined and appeared to be better as 1 part in $10^{7}$.
\end{abstract}

Keywords: Interference refractometer, Laser interferometry, Refractive index

1. Introduction

Laserinterferometry is widely used for accurate measurement of length. The laser interferometer is a basic instrument for the calibration of measuring devices and machine tools and is also used as measuring system in very accurate production- and measuring machines. The accuracy of a laser interferometer is mainly determined by the uncertainties in the light frequency and the refractive index of the environmental air in which the measurement is carried out. The frequency of the laser light can be determined very accurately as described in [3]. Thus the accuracy mainly depends on the accurate determination of the refractive index of air.

This refractive index $(\mathrm{N})$, can be determined basically in two different ways. With the first method, $\mathrm{N}$ is calculated from the measured values of pressure, temperature, humidity and $\mathrm{CO}_{2}$-content using the Edlen formula [2]. Somewhat modified, this equation for the refractive index of air can be written as:

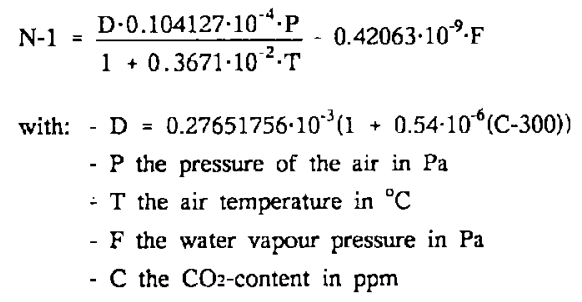

Most of the commercially available laser interferometers use an electronic compensation for changes in $\mathrm{N}$, the so called automatic compensation, based on the above mentioned Edlén formula. The accuracy of this method is at first limited by the model accuracy of the Edlén formula $\left(\frac{\mathrm{dN}}{\mathrm{N}} \leq 5 \cdot 10^{-8}\right)$ and the measuring errors in the determination of $P, T, F$, and $C$ (E.g. an error in the temperature measurement of $0.1 \mathrm{~K}$ introduces a relative error in the calculated refractive index of about $10^{-7}$ ). An other important contribution to the inaccuracy of this method is caused by the contributions of non-modelled constituents in the air. About the effects of these non-modelled constituents we have published earlier in these annals [4]. Taking this all into consideration we may conclude that it will be very difficult to reach a relative accuracy of 1 part in $10^{7}$ with this method.

The second method to determine $\mathbf{N}$ is measuring it with an interference refractometer. With this method $\mathrm{N}$ is calculated from the measured optical path length (OPL $=\mathrm{N} *$ mechanical path length), so the refractive index including the contribution of all constituents is measured. As can be expected this method yields a higher accuracy than Edlén's method. We have proved that it is possible to determine $\mathrm{N}$ with a maximum uncertainty of 5 parts in $10^{8}$ [5]. A disadvantage of this method is the difficult application of most of these instruments. Most of the accurate refractometers $\left(\frac{\mathrm{dN}}{\mathrm{N}} \leq 10^{-7}\right)$ use a sample chamber which has to be evacuated before the measurement can be carried out. So the use of a vactum pump is necessary. Due to the closed structure of most of these instruments, the environmental air has to be pumped smoothly through the sample chamber, this requires a correction for a difference in temperature between the air in the sample chamber and the environmental air and a correction for layers of water molecules on the optical windows. The measurement of this temperature difference and the calculations of the correction will introduce an additional inaccuracy of the refractometer and requires special equipment.

Our goal was to develop a interference refractometer based on a commercially available laser interferometer, which is easy to use, and with a maximum uncertainty of 1 part in $10^{7}$. Therefore the refractometer has to work without vacuum punp and without temperature sensors. For this application we developed a laser interferometer with high stability and a high resolution.

\section{The Plane Mirror Interferometer}

The refractometer is based on a new arrangement of a double-pass plane mirror interferometer, as shown in figure 1 . The interferometer is based on a commercially available laser measuring system using standard optical components. Since a heterodyne two-frequency laser source is used it is necessary to use a polarizing beam-splitter in combination with two quarter-wave plates to obtain the desired beam pattern. The entrance beam which is polarized parallel to the plane of drawing, is depicted as a solid line, while the entrance beam which is polarized perpendicular to the the plane of drawing is depicted as a dashed line. The double-pass principle doubles the resolution of the system compared to a single-pass interferometer. In our configuration this interferometer has a resolution of $\lambda / 800$ (where $\lambda$ is the wavelength of the laser light $(633 \mathrm{~nm})$ ), this corresponds to a displacement resolution of about $0.8 \mathrm{~nm}$. The reflection surface of the bending prism and the beam splitting surface should be parallel within a few arcseconds. The plane mirrors and the comer cube are easy to

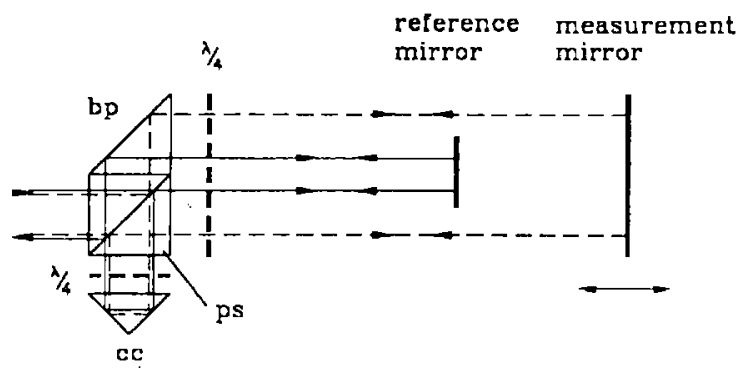

Figure 1. Plane Mirror Interferometer (PS: Polarizing Beams-plitter; BP: Bending Prism; CC: comer cube) 
align. Due to the symmetric beam pattern of this arrangement, the interferometer is insensitive for small rotations (up to a few arcminutes) of the plane mirrors. The interferometer has also a relative low sensitivity for other external influences such as changes in the thermal situation and vibrations. To prove this, several stability measurements have been carried out. For these stability measurements and also in the application with the refractometer, the measurement arm and the reference arm of the interferometer were both reflected on the same plane mirror. The results of one of these measurements are presented in figure 2, which shows a stability within a few counts of $\lambda / 800$.

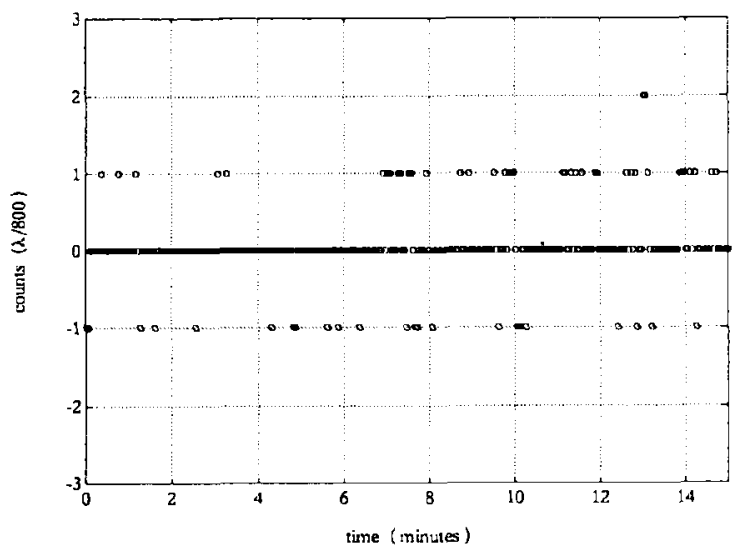

Figure 2. Stability Measurement

\section{The Interference Refractometer.}

The refractometer uses a prismatic chamber with a permanent vacuum inside (see figure 3). Displacing this vacuum chamber perpendicular to the direction of the laser beam causes a change in the path length through the chamber.

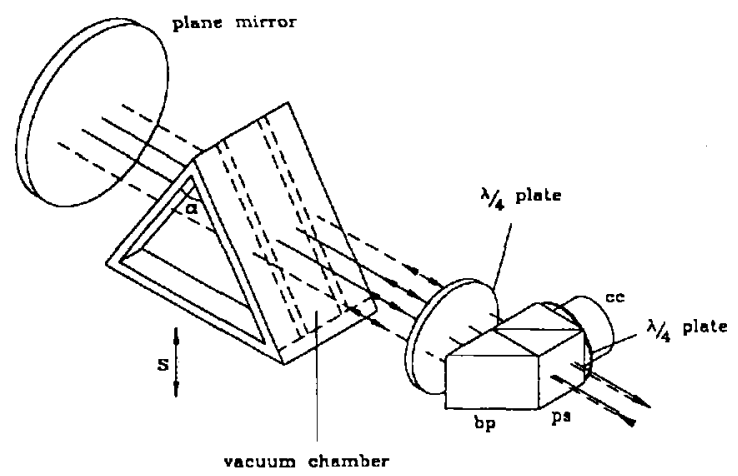

Figure 3. The interference Refractometer

The refractive index can be calculated from the measured optical path length difference, using the following formula:

$N=1+\frac{p \cdot \lambda \cdot r}{r \cdot \Delta L}$

with: $-\Delta \mathrm{L}=2 \cdot \mathrm{S} \cdot \tan (\alpha / 2)$

- S displacement of the vacuum chamber perpendicular to the beams plane $[\mathrm{m}]$

$\alpha$ apex angie of the vacuum chamber

- $p$ the number of pulses counted by the laser measuring system.

- $\lambda_{v}$ the vacuum wavelength of the laser source $[\mathrm{m}]$

- r a resolution factor (in our case $r=800$ )
The beams of the measuring arm as well as the reference arm of the interferometer pass through the windows of the vacuun chamber. So uniform temperature changes of the windows or linear gradients in the temperature of the windows will not affect the measurement results. In order to minimize the effect of non-linear temperature gradients in the windows, a special optical glass is used, which has a low thermo-optical coefficient. This coefficient gives an indication about the change of the optical path length through the glass caused by a change in the temperature of the glass [6].

However, local variations in the optical path length through the windows will introduce unacceptable errors. These local variations can be caused by thickness variations, local variations in the refractive index of the glass due to inhomogeneities in the glass or due to local stress concentrations in the glass. Therefore it is necessary to carry out a correction for these errors. In order to determine the magnitude of this correction the influence of these effects has to be measured. The first method to do this, is to measure the local variations of the optical paths through the windows. This can be realized by measuring the optical path length as a function of the displacement of the vacuum chamber, before the chamber is evacuated. In this case one must be sure that the refractive index inside and outside the chamber will be exactly the same. An other limitation of this method is that the effect of local stress concentrations in the glass, caused by the vacuum pressure can not be inciuded in the correction. The analysis of Birch et al. [1] shows that the absorption of water vapour on a polished glass window has a minor effect on the optical path length through a window. Therefore the influence of vapour absorption on the local variations of the optical path length through the windows will be negligible. A second method to determine the correction is to calibrate the refractometer against another refractometer which has proved to have an higher accuracy. Due to the permanent character of these errors the calibration has to carried out only once. Under laboratory circumstances the method of calculating the refractive index by the Edlen formula can also be used to calibrate the the refractometer. These last two methods more systematic errors can be included in the correction. In our experiments we applied all these methods, as expected the second method yields the highest accuracy. Therefore this method will be applied for the comparison measurements.

The maximum accuracy as well as the resolution of the refractive index measurement are parly limited by the magnitude of the measured optical path length difference. This optical path length difference is mainly determined by the magnitudes of the displacement and the apex angle of the vacuum chamber. As the apex angle of the chamber increases the reflection of laser light on the window surfaces will increases too. The laser measurement system runs out of signal if the apex angle exceers $85^{\circ}$. In the prototype an apex angle of $70^{\circ}$ and a displacement of the chamber of $50 \mathrm{~mm}$ were chosen. This yields a resolution in the measurement of the refractive index of $10^{-8}$. The separate components of the vacuum chamber are made with high precision and are glued together.

To reach the desired accuracy of the refractometer without calibration measurement, some preconditions have to be fulfilled: The apex angle has to be determined with an uncertainty less then 10 arcseconds. Changes of the shape of the chamber caused by the vacuum pressure are negligible. The displacement of the vacuum cell has to be known with an accuracy of $5 \mathrm{\mu m}$. The vacuum chamber has to be well aligned with respect to the laser beams. The alignment error has to be smaller than $0.5^{\circ}$, the rotations of the vactum cell during the displacement must be smaller than a few arcminutes. An accurate determination of the correction for local variations in the optical path length through the windows is essential. In order to reach the highest possible accuracy we determined the correction by one calibration measurement using the TUE-refractometer. After an elaborate analysis of the error sources, the maximum inaccuracy of the refractometer is estimated as 8 parts in $10^{8}$. 
If the refractometer is calibrated, the refractive index can also be calculated by the following formula:

$\mathrm{N}=1+\frac{\mathrm{p}}{\mathrm{p} c} \cdot\left(\mathrm{N}_{\mathrm{c}}-1\right)$

where: $N_{c}=$ Value of the refractive index during the calibration measurement

$p_{c}=$ Number of registered counts during the calibration. measurement

To apply this calculation method, only the following precondition has to be fulfilled: The change in the mechanical path length through the vacuum chamber must be constant for every measurement and the same part of the vacuum chamber has to be used. Consequently a high repeatability of the displacement of the vacuum chamber (S) is required.

\section{Measurements and results}

To evaluate the accuracy of the refractometer, the refractive index of the air is calculated with the Edlen formula and also measured with an other high accuracy interference refractometer, next called the TUE-refractometer [4]. In the comparison set-up the vacuum chamber and the optical components, except the laser source, are placed in an isolated volume, next called the sample volume. The displacement of the vacuum chamber is generated and measured by an Abbe length measuring instrument. The temperature measurement in the sample volume is carried out by resistance thermometers, of the Pt-100 type. For the pressure measurement a calibrated high perfomance quartz crystal pressure transducer is used. The humidity is determined by a wet and dry bulb psychrometer. The $\mathrm{CO}_{2}$-content is measured by an infrared gas analyzer. In our laboratory no other constituents are relevant. Under these laboratory circumstances the refractive index can be calculated with a relative accuracy better than 1 part in $10^{7}$. The TUE-refractometer is connected to the sample volume. The air from the sample volume is circulated smoothly through the TUE-refractometer by a pumping system. The uncertainty of the TUE-refractometer is less than 5 parts in $10^{8}$. The results of these comparisons are presented in figure 4. From these results we can conclude that the maximum uncertainty of the new refractometer is 1 part in $10^{7}$.

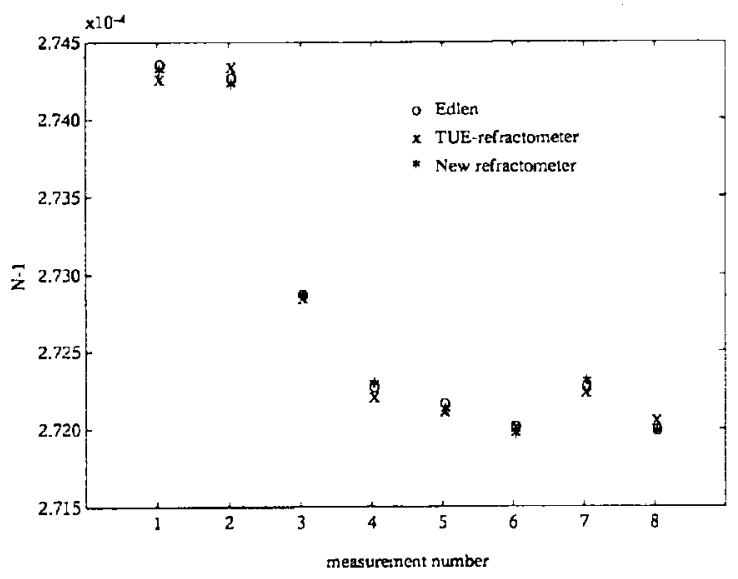

Figure 4. Results of the comparisson measurements

\section{Discussion}

It may be concluded that the new refractometer concept shows good behaviour and can be used for accurate determination of the refractive index of air under industrial conditions. The concept is easy to handle and there is no need for very expensive equipment, especially when the calibration method is used. The concept can probably be improved by using another chamber type, which is in preparation in our laboratories. It is based on a glass tube with an oval cross-section, sealed by optical windows, as depicted in figure 5 . Due to the symmetric arrangement we expect better behaviour and e.g. less influence from local stress effects as a result of glueing the windows. Also the circulation of the air outside the cell is expected to be more realistic with respect to our first concept. Due to time limits it was not possible to present resulis already in this paper.

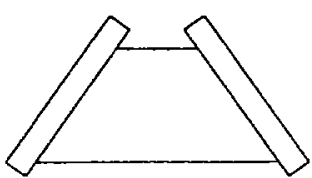

side vleur

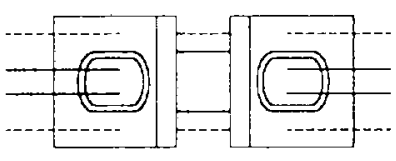

top vieuw with beam arrangement
Figure 5. New design of the vacuum chamber

\section{Acknowledgement}

The authors wish to thank Mr G.J. Theuws for the technical support.

\section{References}

[1] Birch K., Downs M., Ward R., 1988.

The Measurement of Humidity Variations in gases Resulting from the Absorption of Water to Surfaces.

J.Phys. E: Sci. Instrum., No 21, 692-694.

[2] Edlén B., 1966.

The Refractive Index of Air.

Metrologia 2, 71-80.

[3] Schellekens P., 1986.

Absolute Nauwkeurigheid van Technische Laserinterferometers Ph.D. thesis, Eindhoven University of Technology

[4] Schellekens P., Spronck J., van de Pasch E., van der Wolf A., 1986. Design and Results of a New Interference Refractometer Based on a Commercially Available Laserinterferometer. CIRP Annals, Vol 35/1, 387-391.

[5] Schellekens P., Wilkening G., Reinboth F., Downs M., Birch K, Spronck J., 1986.

Measurement of the Refractive Index of Air Using Interference Refractometers.

Metrologia 22, 279-287.

[6] Schott, 1980.

Optisches Glas, Katalog Nr. 3111.

Schott Glaswerke Mainz. 